\title{
Separability of multi-charge black holes in supergravity
}

\author{
Paul Davis ${ }^{1}$ \\ DAMTP, Centre for Mathematical Sciences, \\ University of Cambridge, Wilberforce Rd., \\ Cambridge CB3 0WA, UK
}

\begin{abstract}
In this paper, we show that some five-dimensional rotating black hole solutions of both gauged and ungauged supergravity, with independent rotation parameters and three charges admit separable solutions to the massless Hamilton-Jacobi and Klein-Gordon equations. This allows us to write down a conformal Killing tensor for the spacetime. Conformal Killing tensors obey an equation involving a co-vector field. We find this co-vector field in three specific examples, and also give a general formula for it.
\end{abstract}

\section{Introduction}

It has long been a goal of theoretical physics to understand the origin of the BekensteinHawking entropy of black holes. One potentially fruitful way of attacking this problem is by using holography and, in particular, the AdS/CFT correspondence [1]. From this perspective, it is useful to study asymptotically AdS solutions of gauged supergravity since this theory arises after reducing ten or eleven dimensional supergravity theories on an $S^{n}$. Type IIB supergravity can be reduced on an $S^{5}$ to give five dimensional gauged supergravity in an asymptotically $A d S_{5}$ spacetime. In this case, the boundary theory will then be $\mathcal{N}=4$ superYang-Mills theory which is relatively well understood. Eleven-dimensional supergravity can be reduced on an $S^{7}$, but then the dual theory lives on the boundary of $A d S_{4}$, and is poorly understood. In the $D=5$ case, the $S^{5}$ has isometry group $S O(6)$ which contains $U(1)^{3}$ as a Cartan subgroup. In this paper, we study the hidden symmetries of black hole solutions that are charged under the three $U(1)$ s.

Higher dimensional black holes are currently an area of considerable interest. Higher dimensional, asymptotically flat, Kerr black holes were first found in [2] and the asymptotically (A)dS analogues were found more recently in [3. The five-dimensional Myers-Perry and Kerr(A)dS black holes were shown to possess Killing tensors for arbitrary rotation parameters in [4] and [5] respectively, and in the case where the rotation parameters can take one of two different values, a Killing tensor has been found in all dimensions [6].

\footnotetext{
${ }^{1}$ P.Davis@damtp.cam.ac.uk
} 
Generalisations to rotating black holes with electrical charge have been found. In the case of $D=5$ minimal gauged supergravity, an electrically charged black hole with equal rotation parameters was found in [7] and was shown to possess a reducible Killing tensor in [8]. The solution with unequal rotation parameters was found in [9] and an irreducible Killing tensor for this solution was computed in [10].

Other generalisations have also been discovered. For example, higher dimensional, black holes with NUT charge and a single, non-zero, rotation parameter were found in 11, and Killing tensors for these solutions were found in [12. A class of cohomogeneity-2, rotating, AdS black holes in higher dimensions with a generalisation of the NUT parameter was found in [13] and the Killing tensors were computed in [14, 15. Kerr-NUT-AdS black holes with arbitrary rotation parameters have recently been found in [16.

In this paper, we investigate the separability of some five-dimensional black hole solutions with three charges and two arbitrary rotation parameters. The first black hole solution that we study is the general solution in ungauged $\mathcal{N}=2$ supergravity with three unequal charges that was found in [17. The second solution arises from $S O(6)$ gauged supergravity and has two of its three charges equal [18. The final black hole solution that we study is the supersymmetric, $U(1)^{3}$ black hole found in [19].

All the metrics that we work with have cohomogeneity-2, and the inverse metric has the property that when multiplied by some scalar function, it separates additively into tensors depending on only the two non-trivial coordinates. Unfortunately, the function that we have to multiply the inverse metric by is complicated, so we can only separate the Hamilton-Jacobi equation in the case of null geodesics. This introduces a constant of the motion that is quadratic in the canonical momenta and is due to the existence of a non-trivial conformal Killing tensor. The fact that all of these black hole solutions possess a conformal Killing tensor is unsurprising as they can all be put into the canonical form presented in [20].

Several examples of such black holes are known, and in this paper, we investigate whether they are geodesically integrable. In fact we find that this is only possible if we consider massless particles. This is related to the existence of a non-trivial conformal Killing tensor which obeys the equation $\nabla_{(\mu} K_{\nu \sigma)}=g_{(\mu \nu} V_{\sigma)}$ for some co-vector field $V_{\sigma}$. In this paper we find a general formula for the co-vector field based on fairly relaxed conditions on the spacetime metric which all known three-charge black holes satisfy.

There is some ambiguity in the way in which we separate the Hamilton-Jacobi equation. For the cohomogeneity-2 metrics that we are considering in this paper, the massless HamiltonJacobi equation gives a constant of the motion that is quadratic in the canonical momenta, and can be written in two different ways. Thus, the separation of the Hamilton-Jacobi equation gives rise to two distinct conformal Killing tensors. It turns out that these generate two different co-vectors, which differ by the gradient of a scalar function This can be traced back to the fact that the two Killing tensors differ by a scalar function multiplying the metric. It seems that these co-vector fields act rather like $U(1)$ gauge fields and an antisymmetric field strength tensor can be defined that is invariant under such gauge transformations and perhaps it is this that should be regarded as the entity with physical relevance.

The structure of this paper is as follows. In section 2, we consider the three charge solution of ungauged supergravity found in [17]. We show that the Hamilton-Jacobi equation is separable in the massless case and we show that this is due to the existence of a non-trivial conformal 
Killing tensor which we find. We then compute the associated co-vector field and show that the Klein-Gordon equation is also separable in the massless case. In section 3, we repeat this analysis for the non-extremal black hole solution of $U(1)^{3}$ gauged supergravity with two charges set equal and the third related to it 18. In section 4, we show that the same results hold true for the recently found supersymmetric solution of $U(1)^{3}$ gauged supergravity found in [19]. In section 5 we prove some general results about the form of the associated co-vector fields and show that they control the evolution of a scalar function constructed from the conformal Killing tensor along the worldline of a massive particle. Finally, in section 6 we present our conclusions.

\section{Three-charge black holes from ungauged supergrav- ity}

In this section, we examine the three-charge five-dimensional black holes found in [17. In ungauged supergravity, one can use solution generating techniques to add charges to the neutral Myers-Perry black hole [2. The solution that was found in [17] can be written in the following form:

$$
\begin{aligned}
d s^{2} & =\bar{\Delta}^{\frac{1}{3}}\left[-\frac{\rho^{2}\left(\rho^{2}-2 m\right)}{\bar{\Delta}} d t^{2}+\frac{d r^{2}}{\Delta_{r}(r)}+d \theta^{2}\right. \\
& -\frac{4 m \sin ^{2} \theta}{\bar{\Delta}}\left(\rho^{2}\left(a \operatorname{ch}_{e} \operatorname{ch}_{e 1} \operatorname{ch}_{e 2}-b \operatorname{sh}_{e} \operatorname{sh}_{e 1} \operatorname{sh}_{e 2}\right)+2 m b \operatorname{sh}_{e} \operatorname{sh}_{e 1} \operatorname{sh}_{e 2}\right) d t d \phi \\
& -\frac{4 m \cos ^{2} \theta}{\bar{\Delta}}\left(\rho^{2}\left(b \operatorname{ch}_{e} \operatorname{ch}_{e 1} \operatorname{ch}_{e 2}-a \operatorname{sh}_{e} \operatorname{sh}_{e 1} \operatorname{sh}_{e 2}\right)+2 m a \operatorname{sh}_{e} \operatorname{sh}_{e 1} \operatorname{sh}_{e 2}\right) d t d \psi \\
& +\frac{\sin ^{2} \theta}{\bar{\Delta}}\left(\left(r^{2}+a^{2}+2 m \operatorname{sh}_{e}^{2}\right)\left(\rho^{2}+2 m \operatorname{sh}_{e 1}^{2}\right)\left(\rho^{2}+2 m \operatorname{sh}_{e 2}^{2}\right)\right. \\
& +2 m \sin ^{2} \theta\left\{\left(a^{2} \operatorname{ch}_{e}^{2}-b^{2} \operatorname{sh}_{e}^{2}\right) \rho^{2}+4 m a b \operatorname{sh}_{e} \operatorname{sh}_{e 1} \operatorname{sh}_{e 2} \operatorname{ch}_{e} \operatorname{ch}_{e 1} \operatorname{ch}_{e 2}\right. \\
& \left.\left.-2 m \operatorname{sh}_{e 1}^{2} \operatorname{sh}_{e 2}^{2}\left(a^{2} \operatorname{ch}_{e}^{2}+b^{2} \operatorname{sh}_{e}^{2}\right)-2 m b^{2} \operatorname{sh}_{e}^{2}\left(\operatorname{sh}_{e 1}^{2}+\operatorname{sh}_{e 2}^{2}\right)\right\}\right) d \phi^{2} \\
+ & \frac{\cos ^{2} \theta}{\bar{\Delta}}\left(\left(r^{2}+b^{2}+2 m \operatorname{sh}_{e}^{2}\right)\left(\rho^{2}+2 m \operatorname{sh}_{e 1}^{2}\right)\left(\rho^{2}+2 m \operatorname{sh}_{e 2}^{2}\right)\right. \\
+ & 2 m \cos ^{2} \theta\left\{\left(b^{2} \operatorname{ch}_{e}^{2}-a^{2} \operatorname{sh}_{e}^{2}\right) \rho^{2}+4 m a b \operatorname{sh}_{e} \operatorname{sh}_{e 1} \operatorname{sh}_{e 2} \operatorname{ch}_{e} \operatorname{ch}_{e 1} \operatorname{ch}_{e 2}\right. \\
& \left.\left.-2 m \operatorname{sh}_{e 1}^{2} \operatorname{sh}_{e 2}^{2}\left(b^{2} \operatorname{ch}_{e}^{2}+a^{2} \operatorname{sh}_{e}^{2}\right)-2 m a^{2} \operatorname{sh}_{e}^{2}\left(\operatorname{sh}_{e 1}^{2}+\operatorname{sh}_{e 2}^{2}\right)\right\}\right) d \psi^{2} \\
+ & \frac{4 m \sin ^{2} \theta \cos ^{2} \theta}{\bar{\Delta}}\left(a b\left\{\rho^{2}-2 m\left(\operatorname{sh}_{e 1}^{2} \operatorname{sh}_{e 2}^{2}+\operatorname{sh}_{e}^{2} \operatorname{sh}_{e 1}^{2}+\operatorname{sh}_{e}^{2} \operatorname{sh}_{e 2}^{2}\right)\right\}\right. \\
+ & \left.\left.2 m\left\{\left(a^{2}+b^{2}\right) \operatorname{ch}_{e} \operatorname{ch}_{e 1} \operatorname{ch}_{e 2} \operatorname{sh}_{e} \operatorname{sh}_{e 1} \operatorname{sh}_{e 2}-2 a b \operatorname{sh}_{e}^{2} \operatorname{sh}_{e 1}^{2} \operatorname{sh}_{e 2}^{2}\right\}\right) d \phi d \psi\right]
\end{aligned}
$$

Here, we have used the notation $\operatorname{sh}_{e} \equiv \sinh \delta_{e}, \operatorname{sh}_{e 1} \equiv \sinh \delta_{e 1}$ and $\operatorname{sh}_{e 2} \equiv \sinh \delta_{e 2}$ where $\delta_{e}$, $\delta_{e 1}$ and $\delta_{e 2}$ are the $S O(1,1)$ boosts that were applied to the neutral rotating solution in order to obtain the three charge solution. Also, $\mathrm{ch}_{e} \equiv \cosh \delta_{e}$ and likewise for the other boosts. We have defined the following functions:

$$
\bar{\Delta}=\left(\rho^{2}+2 m \operatorname{sh}_{e}^{2}\right)\left(\rho^{2}+2 m \operatorname{sh}_{e 1}^{2}\right)\left(\rho^{2}+2 m \operatorname{sh}_{e 2}^{2}\right),
$$




$$
\rho^{2}=r^{2}+a^{2} \cos ^{2} \theta+b^{2} \sin ^{2} \theta
$$

and

$$
\Delta_{r}(r)=\frac{\left(r^{2}+a^{2}\right)\left(r^{2}+b^{2}\right)-2 m r^{2}}{r^{2}}
$$

The gauge fields that give rise to the three charges have the following origin: The first one is the Kaluza-Klein gauge field associated with the first compactified direction, the second gauge field comes from the ten-dimensional two form field associated to the first compactified direction, and the field strength of the third gauge field is dual to $H_{\mu \nu \rho}$ which is the threeform field strength of the five-dimensional two-form $B_{\mu \nu}$. The duality between the three and two-form field strengths is given by

$$
H^{\mu \nu \rho}=-\frac{e^{4 \varphi / 3}}{2 ! \sqrt{-g}} \varepsilon^{\mu \nu \rho \lambda \sigma} F_{\lambda \sigma},
$$

where the dilaton field, $\varphi$ is given by the equation

$$
e^{2 \varphi}=\frac{\left(\rho^{2}+2 m \operatorname{sh}_{e}^{2}\right)^{3}}{\bar{\Delta}}
$$

The determinant of this metric is surprisingly simple. We find that

$$
\sqrt{-\operatorname{det} g}=r \sin \theta \cos \theta \bar{\Delta}^{1 / 3}
$$

The inverse metric is also relatively simple and has the non-trivial property that $\bar{\Delta}^{1 / 3} g^{\mu \nu}$ is additively separable as a function of $r$ and a function of $\theta$. We obtain:

$$
\begin{aligned}
\bar{\Delta}^{1 / 3} g^{t t} & =-a^{2} \cos ^{2} \theta-b^{2} \sin ^{2} \theta-r^{2}-2 m\left(\operatorname{sh}_{e}^{2}+\operatorname{sh}_{e 1}^{2}+\operatorname{sh}_{e 2}^{2}\right)-2 m \\
& -\frac{4 m^{2}}{r^{2} \Delta_{r}}\left(r ^ { 2 } \left(1+\operatorname{sh}_{e}^{2}+\operatorname{sh}_{e 1}^{2}+\operatorname{sh}_{e 2}^{2}+\operatorname{sh}_{e}^{2} \operatorname{sh}_{e 1}^{2}+\operatorname{sh}_{e}^{2} \operatorname{sh}_{e 2}^{2}\right.\right. \\
& \left.+\operatorname{sh}_{e 1}^{2} \operatorname{sh}_{e 2}^{2}\right)-\left(a^{2}+b^{2}\right) \operatorname{sh}_{e}^{2} \operatorname{sh}_{e 1}^{2} \operatorname{sh}_{e 2}^{2} \\
& \left.+2 a b \operatorname{ch}_{e} \operatorname{ch}_{e 1} \operatorname{ch}_{e 2} \operatorname{sh}_{e} \operatorname{sh}_{e 1} \operatorname{sh}_{e 2}\right) \\
\bar{\Delta}^{1 / 3} g^{t \phi} & =-\frac{2 m}{r^{2} \Delta_{r}}\left(r^{2}\left(a \operatorname{ch}_{e} \operatorname{ch}_{e 1} \operatorname{ch}_{e 2}-b \operatorname{sh}_{e} \operatorname{sh}_{e 1} \operatorname{sh}_{e 2}\right)\right. \\
& \left.+b\left(2 m-b^{2}\right) \operatorname{sh}_{e} \operatorname{sh}_{e 1} \operatorname{sh}_{e 2}+a b^{2} \operatorname{ch}_{e} \operatorname{ch}_{e 1} \operatorname{ch}_{e 2}\right) \\
\bar{\Delta}^{1 / 3} g^{t \psi} & =\frac{2 m}{r^{2} \Delta_{r}}\left(r^{2}\left(b \operatorname{ch}_{e} \operatorname{ch}_{e 1} \operatorname{ch}_{e 2}-a \operatorname{sh}_{e} \operatorname{sh}_{e 1} \operatorname{sh}_{e 2}\right)\right. \\
& \left.+a\left(2 m-a^{2}\right) \operatorname{sh}_{e} \operatorname{sh}_{e 1} \operatorname{sh}_{e 2}+a^{2} b \operatorname{ch}_{e} \operatorname{ch}_{e 1} \operatorname{ch}_{e 2}\right) \\
\bar{\Delta}^{1 / 3} g^{\phi \phi} & =\frac{1}{\sin ^{2} \theta}-\frac{2 m b^{2}+\left(r^{2}+b^{2}\right)\left(a^{2}-b^{2}\right)}{r^{2} \Delta_{r}}
\end{aligned}
$$




$$
\begin{aligned}
\bar{\Delta}^{1 / 3} g^{\psi \psi} & =\frac{1}{\cos ^{2} \theta}-\frac{2 m a^{2}+\left(r^{2}+a^{2}\right)\left(b^{2}-a^{2}\right)}{r^{2} \Delta_{r}} \\
\bar{\Delta}^{1 / 3} g^{\phi \psi} & =-\frac{2 m a b}{r^{2} \Delta_{r}} \\
\bar{\Delta}^{1 / 3} g^{r r} & =\Delta_{r} \\
\bar{\Delta}^{1 / 3} g^{\theta \theta} & =1
\end{aligned}
$$

\subsection{The Hamilton-Jacobi equation}

The Hamilton-Jacobi equation can be used to generate the equations of the geodesics in this background. If the Hamilton-Jacobi equation is completely separable, then geodesic motion will be integrable. For free, uncharged particles, the Hamiltonian is $H=\frac{1}{2} g^{\mu \nu} p_{\mu} p_{\nu}$, where $\left\{p_{\mu}\right\}$ are the canonical momenta, and the corresponding Hamilton-Jacobi equation is

$$
\frac{\partial S}{\partial \tau}+\frac{1}{2} g^{\mu \nu} \frac{\partial S}{\partial x^{\mu}} \frac{\partial S}{\partial x^{\nu}}=0
$$

where $S$ is Hamilton's principal function and $\tau$ is the parameter along the worldline of the particle. Due to the isometries that are present in this background, $t, \phi$ and $\psi$ all appear linearly in S, so we have:

$$
S=\frac{1}{2} m^{2} \tau-E t+\Phi \phi+\Psi \psi+F(r, \theta)
$$

If we multiply (2.9) by $\bar{\Delta}^{\frac{1}{3}}$, then we can use the additive separability of $\bar{\Delta}^{\frac{1}{3}} g^{\mu \nu}$ to try to fully separate $S$. Substituting (2.10) into (2.9) and multiplying through by $2 \bar{\Delta}^{\frac{1}{3}}$, we obtain

$$
m^{2} \bar{\Delta}^{\frac{1}{3}}+\left(\bar{\Delta}^{\frac{1}{3}} g^{\mu \nu}\right) \frac{\partial S}{\partial x^{\mu}} \frac{\partial S}{\partial x^{\nu}}=0
$$

which will not allow us to separate the $r$ and $\theta$ dependence of $F(r, \theta)$ for arbitrary rotation parameters and charges, due to the complicated form of $\bar{\Delta}^{\frac{1}{3}}$, unless $m^{2}=0$. In the much less general cases where the rotation parameters are equal, $a=b$, or if the charges are all equal, $\delta_{e}=\delta_{e 1}=\delta_{e 2}$, the Hamilton-Jacobi equation will separate for arbitrary $\mathrm{m}^{2}$. Neither of these cases are of interest to us, so we deduce that the Hamilton-Jacobi equation with arbitrary rotation parameters and arbitrary charges will only separate if the mass, $m$, is set to zero. With $m$ set to zero, we can write $F(r, \theta)=S_{r}(r)+S_{\theta}(\theta)$ and then the $\theta$-dependent part of the separated Hamilton-Jacobi equation is

$$
K=-\left(a^{2} \cos ^{2} \theta+b^{2} \sin ^{2} \theta\right) E^{2}+\frac{\Phi^{2}}{\sin ^{2} \theta}+\frac{\Psi^{2}}{\cos ^{2} \theta}+\left(\frac{d S_{\theta}}{d \theta}\right)^{2}
$$

where $K$ is the separation constant. By writing $K=K^{\mu \nu} p_{\mu} p_{\nu}$, where $p_{\mu}$ is the canonical momentum, we find the following expression for the symmetric rank 2 tensor, $K^{\mu \nu}$ :

$$
K^{\mu \nu}=-\left(a^{2} \cos ^{2} \theta+b^{2} \sin ^{2} \theta\right) \delta_{t}^{\mu} \delta_{t}^{\nu}+\frac{\delta_{\phi}^{\mu} \delta_{\phi}^{\nu}}{\sin ^{2} \theta}+\frac{\delta_{\psi}^{\mu} \delta_{\psi}^{\nu}}{\cos ^{2} \theta}+\delta_{\theta}^{\mu} \delta_{\theta}^{\nu}
$$

It is easy to check that $K^{\mu \nu}$ is a conformal Killing tensor, obeying the equation

$$
\nabla_{(\mu} K_{\nu \rho)}=g_{(\mu \nu} V_{\rho)}
$$


where $V_{\mu}$ is some co-vector field associated to the conformal Killing tensor. By contracting indices in equation (2.14), one obtains the following equation for $V^{\mu}$,

$$
V^{\mu}=\frac{2}{7} \nabla_{\nu} K^{\mu \nu}+\frac{1}{7} \nabla^{\mu} K_{\nu}^{\nu}
$$

which gives us the very simple expressions for the (co-)vector field,

$$
V^{\mu}=\frac{\partial_{\theta} \bar{\Delta}^{\frac{1}{3}}}{\bar{\Delta}^{\frac{1}{3}}} \delta_{\theta}^{\mu} \Longleftrightarrow V_{\mu}=\left(\partial_{\theta} \bar{\Delta}^{\frac{1}{3}}\right) \delta_{\mu}^{\theta}
$$

\subsection{The Klein-Gordon equation}

In the same way that we can separate the Hamilton-Jacobi equation in the case where the mass vanishes, we can separate the Klein-Gordon equation in the case of massless particles. This is because we would have to multiply the mass term, $M^{2} \Phi$, by $\bar{\Delta}^{1 / 3}$, which is a complicated function of $r$ and $\theta$.

The massless Klein-Gordon equation can be written in the following form:

$$
\partial_{\mu}\left(\sqrt{-g} g^{\mu \nu} \partial_{\nu} \Phi\right)=0
$$

If we seek a separable solution to this equation, of the form,

$$
\Phi=e^{-i \omega t} e^{i \alpha \phi} e^{i \beta \psi} \Theta(\theta) R(r),
$$

then we obtain the following equation for $\Theta$ :

$$
\frac{1}{\Theta \sin \theta \cos \theta} \frac{d}{d \theta}\left(\sin \theta \cos \theta \frac{d \Theta}{d \theta}\right)-\frac{\alpha^{2}}{\sin ^{2} \theta}-\frac{\beta^{2}}{\cos ^{2} \theta}+\omega^{2}\left(a^{2} \cos ^{2} \theta+b^{2} \sin ^{2} \theta\right)=k
$$

where $k$ is the separation constant. By making the change of variable, $z=\sin ^{2} \theta$, we can rewrite this equation in the following form:

$$
\begin{aligned}
0 & =\frac{d^{2} \Theta}{d z^{2}}+\left(\frac{1}{z}+\frac{1}{z-1}\right) \frac{d \Theta}{d z}-\frac{1}{4 z(1-z)}\left(\frac{\alpha^{2}}{z}+\frac{\beta^{2}}{1-z}\right) \Theta \\
& +\frac{\omega^{2}}{4}\left(\frac{a^{2}}{z}+\frac{b^{2}}{1-z}\right) \Theta-\frac{k \Theta}{4 z(1-z)} .
\end{aligned}
$$

In general, this differential equation has regular singular points at $z=0$ and $z=1$, and an irregular singular point at $z=\infty$ and it can be recast in the form of a confluent Heun equation. In the special case where the rotation parameters are equal, $a=b$, then $z=\infty$ is a regular singular point, and the solution which is regular at $z=0$ is

$$
\Theta(\theta)=A \cos ^{\beta / 2} \theta \sin ^{|\alpha| / 2} \theta F\left(\gamma_{+}, \gamma_{-} ; 1+|\alpha| ; \sin ^{2} \theta\right)
$$

where

$$
\gamma_{ \pm}=\frac{|\alpha|+\beta+1}{2} \pm \frac{1}{2} \sqrt{1-k+a^{2} \omega^{2}} .
$$

The corresponding equation for $R(r)$ is complicated and we shall not examine it here. 


\section{Non-extremal black holes in $D=5, S O(6)$ gauged su- pergravity}

In this section, we consider non-extremal, rotating, black hole solutions of five-dimensional $S O(6)$ gauged supergravity. The solutions that we shall consider have arbitrary rotation parameters, giving the metric cohomogeneity-2, and are charged under all three gauge fields in the $U(1)^{3}$ subgroup of $S O(6)$. The charges are not all independent ${ }^{2}$ as they depend only on a single parameter, $\delta$. The metric to be considered is [18]:

$$
\begin{aligned}
d s^{2} & =H^{-\frac{4}{3}}\left[-\frac{X(r)}{\rho^{2}}\left(d t-a \sin ^{2} \theta \frac{d \phi}{\Xi_{a}}-b \cos ^{2} \theta \frac{d \psi}{\Xi_{b}}\right)^{2}\right. \\
& +\frac{C}{\rho^{2}}\left(\frac{a b}{f_{3}} d t-\frac{b \sin ^{2} \theta}{f_{2}} \frac{d \phi}{\Xi_{a}}-\frac{a \cos ^{2} \theta}{f_{1}} \frac{d \psi}{\Xi_{b}}\right)^{2} \\
& \left.+\frac{Z \sin ^{2} \theta}{\rho^{2}}\left(\frac{a}{f_{3}} d t-\frac{1}{f_{2}} \frac{d \phi}{\Xi_{a}}\right)^{2}+\frac{W \cos ^{2} \theta}{\rho^{2}}\left(\frac{b}{f_{3}} d t-\frac{1}{f_{1}} \frac{d \psi}{\Xi_{b}}\right)^{2}\right] \\
& +H^{\frac{2}{3}}\left(\frac{\rho^{2}}{X} d r^{2}+\frac{\rho^{2}}{\Delta_{\theta}} d \theta^{2}\right),
\end{aligned}
$$

where

$$
\begin{gathered}
f_{1}=r^{2}+a^{2}, \quad f_{2}=r^{2}+b^{2}, \quad f_{3}=f_{1} f_{2}+2 m r^{2} \sinh ^{2} \delta \\
\rho^{2}=r^{2}+a^{2} \cos ^{2} \theta+b^{2} \sin ^{2} \theta, \quad \tilde{\rho}^{2}=\rho^{2}+2 m \sinh ^{2} \delta, \quad H=\frac{\tilde{\rho}^{2}}{\rho^{2}} \\
X(r)=\frac{f_{1} f_{2}}{r^{2}}-2 m+g^{2}\left(f_{1}+2 m \sinh ^{2} \delta\right)\left(f_{2}+2 m \sinh ^{2} \delta\right), \\
C=f_{1} f_{2}\left(X+2 m-\frac{4 m^{2} \sinh ^{4} \delta}{\rho^{2}}\right) \\
Z=-b^{2} C+\frac{f_{2} f_{3}}{r^{2}}\left[f_{3}-g^{2} r^{2}\left(a^{2}-b^{2}\right)\left(f_{1}+2 m \sinh ^{2} \delta\right) \cos ^{2} \theta\right], \\
W=-a^{2} C+\frac{f_{1} f_{3}}{r^{2}}\left[f_{3}+g^{2} r^{2}\left(a^{2}-b^{2}\right)\left(f_{2}+2 m \sinh ^{2} \delta\right) \sin ^{2} \theta\right] \\
\Xi_{a}=1-a^{2} g^{2} \quad \text { and } \Xi_{b}=1-b^{2} g^{2} .
\end{gathered}
$$

The three gauge fields are

$$
A^{1}=A^{2}=\frac{2 m \sinh \delta \cosh \delta}{\tilde{\rho}^{2}}\left(d t-a \sin ^{2} \theta \frac{d \phi}{\Xi_{a}}-b \cos ^{2} \theta \frac{d \psi}{\Xi_{b}}\right)
$$

and

$$
A^{3}=\frac{2 m \sinh ^{2} \delta}{\rho^{2}}\left(b \sin ^{2} \theta \frac{d \phi}{\Xi_{a}}+a \cos ^{2} \theta \frac{d \psi}{\Xi_{b}}\right) .
$$

\footnotetext{
${ }^{2}$ In fact, two of the charges are equal.
} 
There are also three scalar fields: $X_{1}=X_{2}=H^{-\frac{1}{3}}$ and $X_{3}=H^{\frac{2}{3}}$. These scalars are not directly relevant to the study of symmetries on the phase space, but they are important when computing thermodynamic quantities like charge.

The determinant of this metric can readily be computed and we find that,

$$
\sqrt{-\operatorname{det} g}=\frac{r H^{\frac{2}{3}} \rho^{2} \sin \theta \cos \theta}{\Xi_{a} \Xi_{b}} .
$$

The inverse metric has the remarkable property that $\rho^{2} H^{\frac{2}{3}} g^{\mu \nu}$ is additively separable as a function of $r$ and $\theta$. We find that it is given by

$$
\begin{aligned}
\rho^{2} H^{\frac{2}{3}} g^{t t} & =-\frac{\Xi_{a} \Xi_{b}\left(a^{2} \cos ^{2} \theta+b^{2} \sin ^{2} \theta\right)}{\Delta_{\theta}}+\frac{4 a^{2} b^{2} g^{2} m^{2}}{r^{2} X} \sinh ^{4} \delta \\
& -\frac{2 m}{X}\left(a^{2}+b^{2}-a^{2} b^{2} g^{2}+\sinh ^{2} \delta\left(a^{2}+b^{2}-2 a^{2} b^{2} g^{2}\right)\right) \\
& -\frac{2 m a^{2} b^{2}}{r^{2} X}\left(1+2 \sinh ^{2} \delta-\left(a^{2}+b^{2}\right) g^{2} \sinh ^{2} \delta\right) \\
& -\frac{\Xi_{a} \Xi_{b}}{X}\left(f_{1}+2 m \sinh ^{2} \delta\right)\left(f_{2}+2 m \sinh ^{2} \delta\right), \\
\rho^{2} H^{\frac{2}{3}} g^{t \phi} & =\frac{a \Xi_{a} \Xi_{b}}{\Delta_{\theta}}-\frac{a \Xi_{a}}{r^{2} X}\left(\Xi_{b} f_{3}-2 m b^{2}\left[g^{2}\left(f_{1}+b^{2}\right) \sinh ^{2} \delta-\cosh ^{2} \delta+2 m g^{2} \sinh ^{4} \delta\right]\right), \\
\rho^{2} H^{\frac{2}{3}} g^{t \psi} & =\frac{b \Xi_{a} \Xi_{b}}{\Delta_{\theta}}-\frac{b \Xi_{b}}{r^{2} X}\left(\Xi_{a} f_{3}-2 m a^{2}\left[g^{2}\left(f_{2}+a^{2}\right) \sinh ^{2} \delta-\cosh ^{2} \delta+2 m g^{2} \sinh ^{4} \delta\right]\right), \\
\rho^{2} H^{\frac{2}{3}} g^{\phi \phi} & =\frac{\Xi_{a}^{2}\left(\Xi_{b}+\cot ^{2} \theta\right)}{\Delta_{\theta}}-\frac{\Xi_{a}^{2}}{r^{2} X}\left(-g^{2} b^{2} f_{3}+\left(a^{2}-b^{2}\right) f_{2}+2 m b^{2}\left[1-g^{2}\left(f_{1}+b^{2}\right) \sinh ^{2} \delta\right.\right. \\
& \left.\left.-2 m g^{2} \sinh ^{4} \delta\right]\right), \\
\rho^{2} H^{\frac{2}{3}} g^{\psi \psi} & =\frac{\Xi_{b}^{2}\left(\Xi_{a}+\tan ^{2} \theta\right)}{\Delta_{\theta}}-\frac{\Xi_{b}^{2}}{r^{2} X}\left(-g^{2} a^{2} f_{3}+\left(b^{2}-a^{2}\right) f_{1}+2 m a^{2}\left[1-g^{2}\left(f_{2}+a^{2}\right) \sinh ^{2} \delta\right.\right. \\
& \left.\left.-2 m g^{2} \sinh ^{4} \delta\right]\right), \\
\rho^{2} H^{\frac{2}{3}} g^{\phi \psi} & =-\frac{a b g^{2} \Xi_{a} \Xi_{b}}{\Delta_{\theta}}-\frac{a b \Xi_{a} \Xi_{b}}{r^{2} X}\left(-g^{2} f_{3}+2 m\left[1-g^{2}\left(r^{2}+a^{2}+b^{2}\right) \sinh ^{2} \delta\right.\right. \\
& \left.\left.-2 m g^{2} \sinh ^{4} \delta\right]\right), \\
\rho^{2} H^{\frac{2}{3}} g^{\theta \theta} & =\Delta_{\theta}, \\
\rho^{2} H^{\frac{2}{3}} g^{r r} & =X
\end{aligned}
$$

\subsection{The Hamilton-Jacobi equation}

In order to check whether the Hamilton-Jacobi equation is separable or not, we substitute (2.10) into (2.9) and multiply through by $\rho^{2} H^{\frac{2}{3}}$. As in the previous section, if $m \neq 0$, we are unable to separate the Hamilton-Jacobi equation. In the massless case, the $\theta$-dependent part 
of the separated Hamilton-Jacobi equation is

$$
\begin{aligned}
K & =-\frac{\Xi_{a} \Xi_{b}\left(a^{2} \cos ^{2} \theta+b^{2} \sin ^{2} \theta\right)}{\Delta_{\theta}} E^{2}-\frac{2 a \Xi_{a} \Xi_{b}}{\Delta_{\theta}} E \Phi-\frac{2 b \Xi_{a} \Xi_{b}}{\Delta_{\theta}} E \Psi \\
& +\frac{\Xi_{a}^{2}\left(\Xi_{b}+\cot ^{2} \theta\right)}{\Delta_{\theta}} \Phi^{2}+\frac{\Xi_{b}^{2}\left(\Xi_{a}+\tan ^{2} \theta\right)}{\Delta_{\theta}} \Psi^{2}-\frac{2 a b g^{2} \Xi_{a} \Xi_{b}}{\Delta_{\theta}} \Phi \Psi+\Delta_{\theta}\left(\frac{d S_{\theta}}{d \theta}\right)^{2} .
\end{aligned}
$$

The $r$-dependent part is much more complicated, so we restrict our analysis to the simpler case for the moment.

By writing $K=K^{\mu \nu} p_{\mu} p_{\nu}$ where $\left\{p_{\mu}\right\}$ are the canonical momenta, we find:

$$
\begin{aligned}
K^{\mu \nu} & =-\frac{\Xi_{a} \Xi_{b}\left(a^{2} \cos ^{2} \theta+b^{2} \sin ^{2} \theta\right)}{\Delta_{\theta}} \delta_{t}^{\mu} \delta_{t}^{\nu}+\frac{a \Xi_{a} \Xi_{b}}{\Delta_{\theta}}\left(\delta_{t}^{\mu} \delta_{\phi}^{\nu}+\delta_{t}^{\nu} \delta_{\phi}^{\mu}\right)+\frac{b \Xi_{a} \Xi_{b}}{\Delta_{\theta}}\left(\delta_{t}^{\mu} \delta_{\psi}^{\nu}+\delta_{t}^{\nu} \delta_{\psi}^{\mu}\right) \\
& +\frac{\Xi_{a}^{2}\left(\Xi_{b}+\cot ^{2} \theta\right)}{\Delta_{\theta}} \delta_{\phi}^{\mu} \delta_{\phi}^{\nu}+\frac{\Xi_{b}^{2}\left(\Xi_{a}+\tan ^{2} \theta\right)}{\Delta_{\theta}} \delta_{\psi}^{\mu} \delta_{\psi}^{\nu}-\frac{a b g^{2} \Xi_{a} \Xi_{b}}{\Delta_{\theta}}\left(\delta_{\phi}^{\mu} \delta_{\psi}^{\nu}+\delta_{\phi}^{\nu} \delta_{\psi}^{\mu}\right) \\
& +\Delta_{\theta} \delta_{\theta}^{\mu} \delta_{\theta}^{\nu} .
\end{aligned}
$$

This is a conformal Killing tensor and obeys (2.14) with the vector field given by

$$
V^{\mu}=\Delta_{\theta} \frac{\partial_{\theta}\left(\rho^{2} H^{2 / 3}\right)}{\rho^{2} H^{2 / 3}} \delta_{\theta}^{\mu} \Longleftrightarrow V_{\mu}=\partial_{\theta}\left(\rho^{2} H^{2 / 3}\right) \delta_{\mu}^{\theta} .
$$

\subsection{The Klein-Gordon equation}

Again, due to the complicated nature of the function $\rho^{2} H^{\frac{2}{3}}$, we can only find separable solutions to the Klein-Gordon equation in the massless case. Substituting the separable Ansatz (2.18) into the massless Klein-Gordon equation (2.17), we find the following differential equation for $\Theta(\theta)$ :

$$
\begin{aligned}
& \frac{1}{\Theta \sin \theta \cos \theta} \frac{d}{d \theta}\left(\sin \theta \cos \theta \Delta_{\theta} \frac{d \Theta}{d \theta}\right)+\left[\frac{\Xi_{a} \Xi_{b} \omega^{2}\left(a^{2} \cos ^{2} \theta+b^{2} \sin ^{2} \theta\right)}{\Delta_{\theta}}+\frac{2 \alpha \omega a \Xi_{a} \Xi_{b}}{\Delta_{\theta}}\right. \\
& \left.+\frac{2 \beta \omega b \Xi_{a} \Xi_{b}}{\Delta_{\theta}}-\frac{\alpha^{2} \Xi_{a}^{2}\left(\Xi_{b}+\cot ^{2} \theta\right)}{\Delta_{\theta}}+\frac{2 \alpha \beta \Xi_{a} \Xi_{b} a b g^{2}}{\Delta_{\theta}}-\frac{\beta^{2} \Xi_{b}^{2}\left(\Xi_{a}+\tan ^{2} \theta\right)}{\Delta_{\theta}}\right]=k,
\end{aligned}
$$

where $k$ is the separation constant. This equation can be made simpler by letting $z=\sin ^{2} \theta$. Doing this, one finds that

$$
\begin{aligned}
0 & =\frac{d^{2} \Theta}{d z^{2}}+\left(\frac{1}{z}+\frac{1}{z-1}+\frac{1}{z-d}\right) \frac{d \Theta}{d z}+\left(\frac{\Xi_{a} \Xi_{b} \omega^{2} a^{2}}{4 z \Delta_{z}^{2}}+\frac{\Xi_{a} \Xi_{b} \omega^{2} b^{2}}{4(1-z) \Delta_{z}^{2}}\right. \\
& +\frac{a \Xi_{a} \Xi_{b} \alpha \omega}{2 z(1-z) \Delta_{z}^{2}}+\frac{b \Xi_{a} \Xi_{b} \beta \omega}{2 z(1-z) \Delta_{z}^{2}}-\frac{\alpha^{2} \Xi_{a}^{2} \Xi_{b}}{4 z(1-z) \Delta_{z}^{2}}-\frac{\alpha^{2} \Xi_{a}^{2}}{4 z^{2} \Delta_{z}^{2}} \\
& \left.-\frac{\beta^{2} \Xi_{a} \Xi_{b}^{2}}{4 z(1-z) \Delta_{z}^{2}}-\frac{\beta^{2} \Xi_{b}^{2}}{4 z^{2} \Delta_{z}^{2}}+\frac{\alpha \beta \Xi_{a} \Xi_{b} a b g^{2}}{2 z(1-z) \Delta_{z}^{2}}-\frac{k}{4 z(1-z) \Delta_{z}}\right) \Theta,
\end{aligned}
$$

where $d=\Xi_{a} / g^{2}\left(b^{2}-a^{2}\right)$ and $\Delta_{z}=g^{2}\left(a^{2}-b^{2}\right)(z-d)$. This differential equation has four regular singular points and can straightforwardly be put into the form of Heun's equation. If $a=b$, we obtain a differential equation with three regular singular points whose solution can be given in terms of hypergeometric functions. 


\section{Supersymmetric black holes from $D=5, U(1)^{3}$ gauged supergravity}

We consider the most general known supersymmetric solution of $D=5, U(1)^{3}$ gauged supergravity which was recently found in [19]. It has two angular momenta and three charges corresponding to the three Maxwell fields. Rather than being a five parameter theory, the absence of closed timelike curves imposes a constraint, so there are only four free parameters. This leaves us with the following metric:

$$
d s^{2}=-H^{-\frac{2}{3}}\left(d t+\omega_{\phi} d \phi+\omega_{\psi} d \psi\right)^{2}+H^{\frac{1}{3}} h_{m n} d x^{m} d x^{n} .
$$

Here, $H \equiv H_{1} H_{2} H_{3}$ where

$$
H_{I}=1+\frac{\sqrt{\Xi_{a} \Xi_{b}}\left(1+g^{2} \mu_{I}\right)-\Delta_{\theta}}{g^{2} r^{2}} .
$$

We also have the following definitions

$$
\begin{aligned}
& h_{m n} d x^{m} d x^{n}=r^{2}\left\{\frac{d r^{2}}{\Delta_{r}}+\frac{d \theta^{2}}{\Delta_{\theta}}+\frac{\cos ^{2} \theta}{\Xi_{b}^{2}}\left[\Xi_{b}+\cos ^{2} \theta\left(\rho^{2} g^{2}+2(1+b g)(a+b) g\right)\right] d \psi^{2}\right. \\
&+\frac{\sin ^{2} \theta}{\Xi_{a}^{2}}\left[\Xi_{a}+\sin ^{2} \theta\left(\rho^{2} g^{2}+2(1+a g)(a+b) g\right)\right] d \phi^{2} \\
&\left.+\frac{2 \sin ^{2} \theta \cos ^{2} \theta}{\Xi_{a} \Xi_{b}}\left[\rho^{2} g^{2}+2(a+b) g+(a+b)^{2} g^{2}\right] d \psi d \phi\right\} \\
& \Delta_{r}= r^{2}\left[g^{2} r^{2}+(1+a g+b g)^{2}\right], \quad \Delta_{\theta}=1-a^{2} g^{2} \cos ^{2} \theta-b^{2} g^{2} \sin ^{2} \theta \\
& \Xi_{a}= 1-a^{2} g^{2}, \quad \Xi_{b}=1-b^{2} g^{2}, \quad \rho^{2}=r^{2}+a^{2} \cos ^{2} \theta+b^{2} \sin ^{2} \theta \\
& \omega_{\psi}=-\frac{g \cos ^{2} \theta}{r^{2} \Xi_{b}}\left[\rho^{4}+\left(2 r_{m}^{2}+b^{2}\right) \rho^{2}+\frac{1}{2}\left(\beta_{2}-a^{2} b^{2}+\left(a^{2}-b^{2}\right) g^{-2}\right)\right] \\
& \omega_{\phi}=-\frac{g \sin ^{2} \theta}{r^{2} \Xi_{a}}\left[\rho^{4}+\left(2 r_{m}^{2}+a^{2}\right) \rho^{2}+\frac{1}{2}\left(\beta_{2}-a^{2} b^{2}-\left(a^{2}-b^{2}\right) g^{-2}\right)\right] \\
& r_{m}^{2}=(a+b) g^{-1}+a b
\end{aligned}
$$

and

$$
\beta_{2}=\Xi_{a} \Xi_{b}\left(\mu_{1} \mu_{2}+\mu_{2} \mu_{3}+\mu_{3} \mu_{1}\right)-\frac{2 \sqrt{\Xi_{a} \Xi_{b}}\left(1-\sqrt{\Xi_{a} \Xi_{b}}\right)}{g^{2}}\left(\mu_{1}+\mu_{2}+\mu_{3}\right)+\frac{3\left(1-\sqrt{\Xi_{a} \Xi_{b}}\right)^{2}}{g^{4}} .
$$

It seems as if this solution depends on five parameters: $\mu_{1}, \mu_{2}, \mu_{3}, a$ and $b$. However as mentioned above, there is a constraint, so there are only four independent parameters. The constraint is

$$
\mu_{1}+\mu_{2}+\mu_{3}=\frac{1}{\sqrt{\Xi_{a} \Xi_{b}}}\left[2 r_{m}^{2}+3 g^{-2}\left(1-\sqrt{\Xi_{a} \Xi_{b}}\right)\right] .
$$


The determinant of this metric can be written as follows:

$$
\sqrt{-\operatorname{det} g}=\frac{\left(r^{2} H^{\frac{1}{3}}\right) r \sin \theta \cos \theta}{\Xi_{a} \Xi_{b}} .
$$

Using this determinant, it is a long but straightforward calculation to find the inverse metric. We find:

$$
\begin{aligned}
r^{2} H^{\frac{1}{3}} g^{t t} & =-\frac{1}{4 g^{6}}\left[12 g^{4} \Delta_{\theta}+\frac{4 \Xi_{a} \Xi_{b} g^{4}}{\Delta_{\theta}}+\frac{4\left(\Xi_{a} \Xi_{b}\right)^{3 / 2} \mathcal{J}}{r^{4}}-\frac{4 \beta_{2} g^{4}}{r^{4}}-\frac{4 \Xi_{a} \Xi_{b} g^{2}}{r^{2}}\right. \\
& +\frac{1}{r^{2} \Delta_{r}}\left(-g^{8} \beta_{2}^{2}+2 g^{6} \beta_{2}\left(2 r^{2}+a^{2}+b^{2}+a^{2} b^{2} g^{2}\right)\right. \\
& \left.\left.-\left(3+4 g^{2} r_{m}^{2}-\Xi_{a} \Xi_{b}\right)^{2}\right)\right], \\
r^{2} H^{\frac{1}{3}} g^{t \phi} & =-\frac{\Xi_{a}}{2 g}\left[-\frac{2 \Xi_{b}}{\Delta_{\theta}}+\frac{2 \Xi_{b} r^{2}+a^{2} \Xi_{b}-4 b^{2} g^{2} r_{m}^{2}-2 b^{4} g^{2}-b^{2}-\beta_{2} g^{2}}{\Delta_{r}}\right] \\
r^{2} H^{\frac{1}{3}} g^{t \psi} & =-\frac{\Xi_{b}}{2 g}\left[-\frac{2 \Xi_{a}}{\Delta_{\theta}}+\frac{2 \Xi_{a} r^{2}+b^{2} \Xi_{a}-4 a^{2} g^{2} r_{m}^{2}-2 a^{4} g^{2}-a^{2}-\beta_{2} g^{2}}{\Delta_{r}}\right], \\
r^{2} H^{\frac{1}{3}} g^{\phi \psi} & =\Xi_{a} \Xi_{b}\left[\frac{r^{2}}{\Delta_{r}}-\frac{1}{\Delta_{\theta}}\right] \\
r^{2} H^{\frac{1}{3}} g^{\phi \phi} & =\Xi_{a}^{2}\left[\frac{\cot ^{2} \theta}{\Delta_{\theta}}+\frac{r^{2}}{\Delta_{r}}\right] \\
r^{2} H^{\frac{1}{3}} g^{\psi \psi} & =\Xi_{b}^{2}\left[\frac{\tan ^{2} \theta}{\Delta_{\theta}}+\frac{r^{2}}{\Delta_{r}}\right] \\
r^{2} H^{\frac{1}{3}} g^{r r} & =\Delta_{r}, \\
r^{2} H^{\frac{1}{3}} g^{\theta \theta} & =\Delta_{\theta} \cdot
\end{aligned}
$$

This shows that $r^{2} H^{\frac{1}{3}} g^{\mu \nu}$ is additively separable as a function of $r$ and $\theta$.

The constant $\mathcal{J}$ used above in the $g^{\text {tt }}$ component is defined by the equation:

$$
\mathcal{J}=\left(1+g^{2} \mu_{1}\right)\left(1+g^{2} \mu_{2}\right)\left(1+g^{2} \mu_{3}\right) \text {. }
$$

\subsection{The Hamilton-Jacobi equation}

As in the previous two examples, the Hamilton-Jacobi equation (2.9) is only completely separable ${ }^{3}$, for arbitrary charges and rotation parameters if $m^{2}=0$. This is due to the fact that $H^{\frac{1}{3}}$ is not a simple function of $r$ and $\theta$.

\footnotetext{
${ }^{3}$ That is, we can write the $F(r, \theta)$ part of (2.10) as $F(r, \theta)=S_{r}(r)+S_{\theta}(\theta)$.
} 
If $m^{2}=0$, then the $\theta$-separation equation is

$$
\begin{aligned}
K & =-\frac{1}{4 g^{6}}\left[12 g^{4} \Delta_{\theta}+\frac{4 \Xi_{a} \Xi_{b} g^{4}}{\Delta_{\theta}}\right] E^{2}-\frac{2 \Xi_{a} \Xi_{b}}{g \Delta_{\theta}} E(\Phi+\Psi) \\
& +\frac{\left(\Xi_{a} \cot \theta \Phi-\Xi_{b} \tan \theta \Psi\right)^{2}}{\Delta_{\theta}}+\Delta_{\theta}\left(\frac{d S_{\theta}}{d \theta}\right)^{2} .
\end{aligned}
$$

From the $\theta$ separation equation, we can extract a second order tensor, $K^{\mu \nu}$, defined by $K=$ $K^{\mu \nu} p_{\mu} p_{\nu}$ where the $p_{\mu}$ are the canonical momenta. If this complete separation was valid for all $m^{2}$, then $K^{\mu \nu}$ would be a Killing tensor, and would satisfy $\nabla_{(\mu} K_{\nu \sigma)}=0$. However, as the Hamilton-Jacobi equation only appears to separate if $m^{2}=0$, then we would only expect $K^{\mu \nu}$ to be a conformal Killing tensor, which would satisfy $\nabla_{(\mu} K_{\nu \sigma)}=g_{(\mu \nu} V_{\sigma)}$ for some co-vector field $V_{\sigma}$.

We find that $K^{\mu \nu}$ is given by:

$$
\begin{aligned}
K^{\mu \nu} & =-\frac{1}{4 g^{6}}\left[12 g^{4} \Delta_{\theta}+\frac{4 \Xi_{a} \Xi_{b} g^{4}}{\Delta_{\theta}}\right] \delta_{t}^{\mu} \delta_{t}^{\nu}+\frac{\Xi_{a} \Xi_{b}}{g \Delta_{\theta}}\left(\delta_{t}^{\mu} \delta_{\phi}^{\nu}+\delta_{t}^{\nu} \delta_{\phi}^{\mu}+\delta_{t}^{\mu} \delta_{\psi}^{\nu}+\delta_{t}^{\nu} \delta_{\psi}^{\mu}\right) \\
& +\frac{\Xi_{a}^{2} \cot ^{2} \theta}{\Delta_{\theta}} \delta_{\phi}^{\mu} \delta_{\phi}^{\nu}+\frac{\Xi_{b}^{2} \tan ^{2} \theta}{\Delta_{\theta}} \delta_{\psi}^{\mu} \delta_{\psi}^{\nu}-\frac{\Xi_{a} \Xi_{b}}{\Delta_{\theta}}\left(\delta_{\phi}^{\mu} \delta_{\psi}^{\nu}+\delta_{\phi}^{\nu} \delta_{\psi}^{\mu}\right)+\Delta_{\theta} \delta_{\theta}^{\mu} \delta_{\theta}^{\nu} .
\end{aligned}
$$

In order to find $V_{\sigma}$, we can use equation (2.15) with the result:

$$
V^{\mu}=\frac{\partial_{\theta} H^{\frac{1}{3}}}{H^{\frac{1}{3}}} \Delta_{\theta} \delta_{\theta}^{\mu} \Longleftrightarrow V_{\mu}=\partial_{\theta}\left(r^{2} H^{\frac{1}{3}}\right) \delta_{\mu}^{\theta} .
$$

\subsection{The Klein-Gordon equation}

As in the previous cases, we are unable to separate the Klein-Gordon equation in the massive case due to the nature of the function $H^{\frac{1}{3}}$. Taking the massless Klein-Gordon equation (2.17) and substituting into it the separable Ansatz (2.18), we find the following equation for $\Theta(\theta)$ :

$$
\begin{aligned}
\frac{1}{\Theta \sin \theta \cos \theta} \frac{d}{d \theta}\left(\sin \theta \cos \theta \Delta_{\theta} \frac{d \Theta}{d \theta}\right)+\left[\frac{\omega^{2}}{4 g^{2}}\left(\frac{\Xi_{a} \Xi_{b}}{\Delta_{\theta}}+3 \Delta_{\theta}\right)+\frac{2(\alpha+\beta) \omega \Xi_{a} \Xi_{b}}{g \Delta_{\theta}}\right. \\
\left.-\frac{\alpha^{2} \Xi_{a}^{2} \cot ^{2} \theta}{\Delta_{\theta}}+\frac{2 \alpha \beta \Xi_{a} \Xi_{b}}{\Delta_{\theta}}-\frac{\beta^{2} \Xi_{b}^{2} \tan ^{2} \theta}{\Delta_{\theta}}\right]=k,
\end{aligned}
$$

where $k$ is the separation constant. The analytic structure of this equation can be seen more easily if we make the standard change of variable $z=\sin ^{2} \theta$. This gives us

$$
\begin{aligned}
0 & =\frac{d^{2} \Theta}{d z^{2}}+\left(\frac{1}{z}+\frac{1}{z-1}+\frac{1}{z-d}\right) \frac{d \Theta}{d z}+\left[\frac{\omega^{2}}{4 g^{2}}\left(\frac{\Xi_{a} \Xi_{b}}{z(1-z) \Delta_{z}^{2}}+\frac{3}{z(1-z)}\right)\right. \\
& \left.+\frac{(\alpha+\beta) \omega \Xi_{a} \Xi_{b}}{2 g z(1-z) \Delta_{z}^{2}}-\frac{\alpha^{2} \Xi_{a}^{2}}{4 z^{2} \Delta_{z}^{2}}-\frac{\beta^{2} \Xi_{b}^{2}}{4(1-z)^{2} \Delta_{z}^{2}}+\frac{\alpha \beta \Xi_{a} \Xi_{b}}{2 z(1-z) \Delta_{z}^{2}}-\frac{k}{4 z(1-z) \Delta_{z}}\right] \Theta,
\end{aligned}
$$

where $d=\Xi_{a} / g^{2}\left(b^{2}-a^{2}\right)$ and $\Delta_{z}=g^{2}\left(a^{2}-b^{2}\right)(z-d)$. This differential equation has four regular singular points and can be put in the form of Heun's equation. In the case where the rotation parameters are equal, this differential equation has regular singular points at $z=0$, $z=1$ and $z=\infty$ and the solution can again be given in terms of hypergeometric functions. 


\section{General Results}

A rank 2, conformal Killing tensor, $K^{\mu \nu}$ in $D$ dimensions is a symmetric tensor that generates a non-trivial symmetry on the phase space for massless particles. From the perspective of integrability, a non-trivial conformal Killing tensor gives rise to an extra constant of the motion that is quadratic in the canonical momenta. Together with the constants of motion due to the Killing vectors and conformal Killing vectors of the spacetime, there are enough constants of the motion to completely describe the geodesic motion of massless particles giving rise to an integrable system. Conformal Killing tensors obey the equation

$$
\nabla_{(\mu} K_{\nu \rho)}=g_{(\mu \nu} V_{\rho)}
$$

for some co-vector field $V_{\rho}$. If $V_{\rho}=0$, then $K^{\mu \nu}$ is a Killing tensor. By contracting (5.1) with $g^{\mu \nu}$, one obtains an equation for the associated vector field, $V^{\mu}$ :

$$
V^{\mu}=\frac{2}{D+2} \nabla_{\nu} K^{\mu \nu}+\frac{1}{D+2} \nabla^{\mu} K_{\nu}^{\nu}
$$

Clearly, any rank two tensor of the form $K^{\mu \nu}=\Lambda\left(x^{\rho}\right) g^{\mu \nu}$ will obey (5.1) with $V_{\mu}=\nabla_{\mu} \Lambda$, so in that sense, any spacetime possesses an infinite family of conformal Killing tensors just as any constant multiplying the inverse spacetime metric will be a Killing tensor. However, in certain circumstances, one finds second rank tensors that obey (5.1), but which are not conformal to the spacetime (inverse) metric. These are non-trivial conformal Killing tensors. Given a non-trivial conformal Killing tensor, one can define an equivalence class so that two conformal Killing tensors are in the same equivalence class if they differ by some function multiplying the inverse metric, that is:

$$
K^{\mu \nu} \sim L^{\mu \nu} \Longleftrightarrow K^{\mu \nu}=L^{\mu \nu}+\Lambda\left(x^{\rho}\right) g^{\mu \nu}
$$

for some function, $\Lambda$. If $V_{\mu}$ and $U_{\mu}$ are the co-vectors associated to $K^{\mu \nu}$ and $L^{\mu \nu}$ respectively, then

$$
V_{\mu}=U_{\mu}+\nabla_{\mu} \Lambda
$$

So, the associated vector fields transform within each equivalence class like $U(1)$ gauge fields which implies that we can define a gauge-invariant field strength on each equivalence class of conformal Killing tensors:

$$
F_{\mu \nu}=\nabla_{\mu} V_{\nu}-\nabla_{\nu} V_{\mu}
$$

We shall now prove a lemma that shows, under reasonable assumptions, that cohomogeneity 2 metrics where the inverse metric can be additively separated after multiplying through by some function admit a conformal Killing tensor, and we show that the associated (co-)vector takes the expected form. We shall prove this result in five-dimensions, but generalisations to higher dimensions follow trivially.

Lemma: Let $g_{\mu \nu}$ be the metric on a five-dimensional spacetime with coordinates $t, r, \theta, \phi$ and $\psi$ and let the metric have cohomogeneity 2 so that $g_{\mu \nu} \equiv g_{\mu \nu}(r, \theta)$. We shall also assume that the $(r, \theta)$ part of the metric can be put into the diagonal form:

$$
d s_{r, \theta}^{2}=\Omega(r, \theta)\left(\Theta^{2}(\theta) d \theta^{2}+R^{2}(r) d r^{2}\right)
$$


and suppose that $\Omega(r, \theta) g^{\mu \nu}$ can be written as a tensor depending only on $r$ plus a tensor depending only on $\theta^{4}$ :

$$
\Omega(r, \theta) g^{\mu \nu}=P^{\mu \nu}(\theta)+S^{\mu \nu}(r) .
$$

Then, $P^{\mu \nu}(\theta)$ and $S^{\mu \nu}(r)$ are conformal Killing tensors with corresponding vector fields

$$
V^{\mu}=\left(\partial_{\theta} \Omega\right) g^{\theta \theta} \delta_{\theta}^{\mu}
$$

and

$$
U^{\mu}=\left(\partial_{r} \Omega\right) g^{r r} \delta_{r}^{\mu}
$$

respectively.

Proof: Let $P^{\mu \nu}(\theta)$ be defined by equation (5.7). We shall evaluate (5.1) with all three indices raised in order to minimise calculational effort.

$$
\begin{aligned}
\nabla^{(\mu} P^{\nu \rho)} & =g^{\sigma(\mu} \partial_{\sigma} P^{\nu \rho)}+g^{\sigma(\mu} \Gamma_{\sigma \tau}^{\nu} P^{\rho) \tau}+g^{\sigma(\mu} \Gamma_{\sigma \tau}^{\rho} P^{\nu) \tau} \\
& =g^{\theta(\mu} \partial_{\theta} P^{\nu \rho)}+\frac{1}{2} g^{\sigma(\mu} g^{\nu|\alpha|}\left(g_{\sigma \alpha, \tau}+g_{\tau \alpha, \sigma}-g_{\sigma \tau, \alpha}\right) P^{\rho) \tau} \\
& +\frac{1}{2} g^{\sigma(\mu} g^{\rho|\alpha|}\left(g_{\sigma \alpha, \tau}+g_{\tau \alpha, \sigma}-g_{\sigma \tau, \alpha}\right) P^{\nu) \tau} \\
& =g^{\theta \theta} \delta_{\theta}^{(\mu} \partial_{\theta}\left(\Omega g^{\nu \rho)}\right)+\frac{1}{2} g^{\sigma(\mu} g^{\nu|\alpha|} g_{\sigma \alpha, \theta} P^{\rho) \theta}+\frac{1}{2} g^{\sigma(\mu} g^{\rho|\alpha|} g_{\sigma \alpha, \theta} P^{\nu) \theta} \\
& =g^{\theta \theta} \delta_{\theta}^{(\mu} g^{\nu \rho)} \partial_{\theta} \Omega+\Omega g^{\theta \theta} \delta_{\theta}^{(\mu} \partial_{\theta} g^{\nu \rho)}+\Omega g^{\theta \theta} \delta_{\theta}^{(\mu} g^{\sigma(\mu} g^{\nu|\alpha|} \delta_{\theta}^{\rho)} g_{\sigma \alpha, \theta} \\
& =g^{(\mu \nu}\left(\delta_{\theta}^{\rho)} g^{\theta \theta} \partial_{\theta} \Omega\right) .
\end{aligned}
$$

In performing this calculation, we have made use of the facts that $P^{r \mu}=0, P^{\mu \theta}=P^{\theta \theta} \delta_{\theta}^{\mu}$ as the $(r, \theta)$ part of the metric is diagonal and $\partial_{\mu} P^{\nu \rho}=\delta_{\mu}^{\theta} \partial_{\theta} P^{\nu \rho}$ as $P^{\mu \nu}$ is a function of $\theta$ only. The $|\alpha|$ notation means that the index $\alpha$ should not be included in the symmetrisation of the indices.

Thus, we have proved that $P^{\mu \nu}(\theta)$ is a conformal Killing tensor. Moreover, we have shown that the associated vector field is $V^{\mu}=\delta_{\theta}^{\mu} g^{\theta \theta} \partial_{\theta} \Omega$ and the co-vector field is $V_{\mu}=\delta_{\mu}^{\theta} \partial_{\theta} \Omega$. The corresponding result from the $r$-dependent conformal Killing tensor, $S^{\mu \nu}$ is $U_{\mu}=\delta_{\mu}^{r} \partial_{r} \Omega$.

It is immediately apparent that the two conformal Killing tensors, $P^{\mu \nu}$ and $S^{\mu \nu}$, have different vector fields associated to them. However, up to a sign, they give rise to the same constant of the motion when one separates the Hamilton-Jacobi equation. To see this, we consider the Hamilton-Jacobi equation,

$$
\frac{\partial S}{\partial \tau}+\frac{1}{2} g^{\mu \nu} \frac{\partial S}{\partial x^{\mu}} \frac{\partial S}{\partial x^{\nu}}=0
$$

and we seek a separable solution to it, of the form ${ }^{5}$

$$
S=\frac{1}{2} m^{2} \tau-E t+\Phi \phi+\Psi \psi+S_{r}(r)+S_{\theta}(\theta) .
$$

\footnotetext{
${ }^{4}$ The first two of the solutions that we studied can be put into the canonical form given in [20] which possesses all of these properties. The third solution that we studied [19] also matches these criteria. Therefore, these assumptions on the properties of the metric are reasonable.

${ }^{5}$ The black hole spacetimes that we have considered have Killing vectors $\partial_{t}, \partial_{\phi}$ and $\partial_{\psi}$, so these coordinates appear linearly in Hamilton's principal function.
} 
Inserting this into (5.11), and multiplying through by $2 \Omega$, we find:

$$
\begin{aligned}
0 & =m^{2} \Omega+(-E)^{2}\left(P^{t t}+S^{t t}\right)+2(-E) \Phi\left(P^{t \phi}+S^{t \phi}\right)+2(-E) \Psi\left(P^{t \psi}+S^{t \psi}\right) \\
& +\Phi^{2}\left(P^{\phi \phi}+S^{\phi \phi}\right)+2 \Psi \Phi\left(P^{\phi \psi}+S^{\phi \psi}\right)+\Psi^{2}\left(P^{\psi \psi}+S^{\psi \psi}\right) \\
& +P^{\theta \theta}\left(\frac{d S_{\theta}}{d \theta}\right)^{2}+S^{r r}\left(\frac{d S_{r}}{d r}\right)^{2} .
\end{aligned}
$$

The function $\Omega$ is not generally the sum of a function of $r$ and a function of $\theta$, so the $r$ and $\theta$ dependence can only be separated in the massless case, that is if $m^{2}=0$. Given a separable solution to the Hamilton-Jacobi equation, we can deduce the geodesic equations up to quadratures. Having set $m^{2}=0$, the $r$ and $\theta$ dependence can be separated as follows:

$$
\begin{aligned}
K & =(-E)^{2} P^{t t}+2(-E) \Phi P^{t \phi}+2(-E) \Psi P^{t \psi} \\
& +\Phi^{2} P^{\phi \phi}+2 \Psi \Phi P^{\phi \psi}+\Psi^{2} P^{\psi \psi}+P^{\theta \theta}\left(\frac{d S_{\theta}}{d \theta}\right)^{2}
\end{aligned}
$$

and

$$
\begin{aligned}
-K & =(-E)^{2} S^{t t}+2(-E) \Phi S^{t \phi}+2(-E) \Psi S^{t \psi} \\
& +\Phi^{2} S^{\phi \phi}+2 \Psi \Phi S^{\phi \psi}+\Psi^{2} S^{\psi \psi}+S^{r r}\left(\frac{d S_{r}}{d r}\right)^{2} .
\end{aligned}
$$

Letting $K=K^{\mu \nu} p_{\mu} p_{\nu}$, where $K^{\mu \nu}$ is the conformal Killing tensor, we have a choice. We can choose $K^{\mu \nu}=P^{\mu \nu}$ or $K^{\mu \nu}=-S^{\mu \nu}$. So, the two possible conformal Killing tensors differ by $\Omega g^{\mu \nu}$, and thus lie in the same equivalence class. This also explains the difference in the associated vector fields.

Independently of the choice of conformal Killing tensor, the only non-zero components of the associated field strength, $F_{\mu \nu}$, are $F_{r \theta}=-F_{\theta r}=\partial_{r} \partial_{\theta} \Omega$. In the case where $\Omega=f(r)+g(\theta)$, this field strength vanishes which is unsurprising because in that case, we would have a Killing tensor and not a conformal Killing tensor.

\subsection{Relation to Killing tensors}

Here we consider the case when $\Omega(r, \theta)$ can be written as a function of $r$ plus a function of $\theta$. This will give rise to Killing tensors rather than conformal Killing tensors. Let $\Omega(r, \theta)=$ $f(\theta)+h(r)$. Then the equation for the separation of the inverse metric can be written as:

$$
(f(\theta)+h(r)) g^{\mu \nu}=P^{\mu \nu}(\theta)+S^{\mu \nu}(r) .
$$

As it stands, $P^{\mu \nu}$ and $S^{\mu \nu}$ are conformal Killing tensors, but it is easy to show that

$$
P^{\prime \mu \nu}=P^{\mu \nu}(\theta)-f(\theta) g^{\mu \nu} \quad \text { and } \quad S^{\prime \mu \nu}=S^{\mu \nu}(r)-h(r) g^{\mu \nu}
$$

are Killing tensors. In fact, it is very easy to see that $P^{\prime \mu \nu}=-S^{\prime \mu \nu}$. 


\subsection{Relevance of the vector field}

We have seen that the constant of motion that arises in the separation of the massless Hamilton-Jacobi equation can be expressed in terms of a conformal Killing tensor, and that in reality, there are two possible conformal Killing tensors that will give rise to the same constant of motion. As we discussed earlier, these two conformal Killing tensors have different vector fields associated to them, so this raises the question as to what, if any, physical relevance can be attached to these vector fields.

If we compute the Poisson bracket of $K=K^{\mu \nu} p_{\mu} p_{\nu}$ with the Hamiltonian, $H=g^{\mu \nu} p_{\mu} p_{\nu}$, we find the rather simple result

$$
\{K, H\}=2 H\left(V^{\mu} p_{\mu}\right),
$$

where $V^{\mu}$ is the vector field associated to the conformal Killing tensor, $K^{\mu \nu}$, and $p_{\mu}$ are the canonical momenta.

The Poisson bracket of a function with the Hamiltonian describes the evolution of the function along the particle worldline:

$$
\frac{d f}{d \tau}=\{f, H\}
$$

so we see that on massless geodesics (where $H=0$ ) $K$ is constant as expected. We also see the importance of the vector field $V^{\mu}$. On massive paths, it controls the evolution of the scalar quantity $K$ as you go along the particle worldline. Performing the necessary integration, we obtain

$$
K(\tau)=K_{0}-m^{2} \int_{0}^{\tau} V^{\mu} p_{\mu} d \tau^{\prime} .
$$

The scalar $K$ differs from the scalar obtained from the other conformal Killing tensor by $m^{2} \Omega(\tau)$ in the massive case.

\section{Conclusions}

In this paper, we have studied three black hole solutions of five dimensional supergravity with three charges and independent rotation parameters. In each case, we found that the inverse metric multiplied by some function can be separated, additively, as a function of the radial variable, $r$, and an angular variable, $\theta$. However, the function that we multiply the inverse metric by takes a rather unpleasant form unless we simplify matters by taking equal rotation parameters or equal charges. This means that the Hamilton-Jacobi equation which governs geodesic flow on the cotangent bundle cannot be separated apart from in the case of massless particles. The separability in the massless case is linked to the existence of a rank two, conformal Killing tensor which we found in all cases. A rank two, conformal Killing tensor obeys an equation that involves a (co-)vector field. For the black hole solutions under investigation here, we have computed these (co-)vector fields and their rather simple expressions have allowed us to suggest and prove a general form.

The separation of the Hamilton-Jacobi equation involves the introduction of a separation constant which appears in both the $r$ and $\theta$ separation equations. Therefore, one can extract two conformal Killing tensors for each black hole, although they differ by a function mul-

tiplying the inverse metric. The associated (co-)vector fields then differ by the gradient of 
the aforementioned function. The physical relevance of these (co-)vectors can be seen when considering the evolution of the quantity $K^{\mu \nu} p_{\mu} p_{\nu}$ along a massive particle worldline.

Finally, we have showed that the Klein-Gordon equation is also separable in the massless case for all three black holes considered here.

\section{Acknowledgements}

The author would like to thank Gary Gibbons, Hari K. Kunduri, James Lucietti and Chris Pope for many useful discussions and comments on this manuscript.

\section{References}

[1] J. M. Maldacena, "The large N limit of superconformal field theories and supergravity," Adv. Theor. Math. Phys. 2 (1998) 231, hep-th/9711200.

[2] R. C. Myers and M. J. Perry, "Black holes in higher dimensional space-times," Annals Phys. 172 (1986) 304.

[3] G. W. Gibbons, H. Lü, D. N. Page and C. N. Pope, "The general Kerr-de Sitter metrics in all dimensions," J. Geom. Phys. 53 (2005) 49, hep-th/0404008

[4] V. P. Frolov and D. Stojkovic, "Particle and light motion in a space-time of a fivedimensional rotating black hole," Phys. Rev. D 68 (2003) 064011, gr-qc/0301016.

[5] H. K. Kunduri and J. Lucietti, "Integrability and the Kerr-(A)dS black hole in five dimensions," Phys. Rev. D 71 (2005) 104021, hep-th/0502124.

[6] M. Vasudevan and K. A. Stevens, "Integrability of particle motion and scalar field propagation in Kerr-(Anti) de Sitter black hole spacetimes in all dimensions," Phys. Rev. D 72 (2005) 124008, gr-qc/0507096.

[7] M. Cvetič, H. Lü and C. N. Pope, "Charged Kerr-de Sitter black holes in five dimensions," Phys. Lett. B 598 (2004) 273, hep-th/0406196.

[8] H. K. Kunduri and J. Lucietti, "Notes on non-extremal, charged, rotating black holes in minimal $D=5$ gauged supergravity," Nucl. Phys. B 724 (2005) 343, hep-th/0504158.

[9] Z. W. Chong, M. Cvetič, H. Lü and C. N. Pope, "General non-extremal rotating black holes in minimal five-dimensional gauged supergravity," Phys. Rev. Lett. 95 (2005) 161301, hep-th/0506029.

[10] P. Davis, H. K. Kunduri and J. Lucietti, "Special symmetries of the charged Kerr-AdS black hole of $D=5$ minimal gauged supergravity," Phys. Lett. B 628 (2005) 275, hep-th/0508169.

[11] D. Klemm, "Rotating black branes wrapped on Einstein spaces," JHEP 9811 (1998) 019, hep-th/9811126 
[12] Z. W. Chong, G. W. Gibbons, H. Lü and C. N. Pope, "Separability and Killing tensors in Kerr-Taub-NUT-de Sitter metrics in higher dimensions," Phys. Lett. B 609 (2005) 124, hep-th/0405061.

[13] W. Chen, H. Lü and C. N. Pope, "Kerr-de Sitter black holes with NUT charges," to appear in Nucl. Phys. B, hep-th/0601002.

[14] W. Chen, H. Lü and C. N. Pope, "Separability in cohomogeneity-2 Kerr-NUT-AdS metrics," JHEP 04 (2006) 008, hep-th/0602084.

[15] P. Davis, "A Killing tensor for higher dimensional Kerr-AdS black holes with NUT charge," Class. Quantum Grav. 23 (2006) 3607, hep-th/0602118.

[16] W. Chen, H. Lü and C. N. Pope, "General Kerr-NUT-AdS metrics in all dimensions,"hep-th/0604125.

[17] M. Cvetič and D. Youm, "General rotating five dimensional black holes of toroidally compactified heterotic string," Nucl. Phys. B 476 (1996) 118, hep-th/9603100.

[18] Z. W. Chong, M. Cvetič, H. Lü and C. N. Pope, "Five-dimensional gauged supergravity black holes with independent rotation parameters," Phys. Rev. D 72 (2005) 041901, hep-th/0505112.

[19] H. K. Kunduri, J. Lucietti and H. S. Reall, "Supersymmetric multi-charge $A d S_{5}$ black holes," JHEP 04 (2006) 036, hep-th/0601156.

[20] Z. W. Chong, M. Cvetič, H. Lü and C. N. Pope, "Non-extremal rotating black holes in five-dimensional gauged supergravity," hep-th/0606213.

[21] J. C. Breckenridge, R. C. Myers, A. W. Peet and C. Vafa, "D-branes and spinning black holes," Phys. Lett. B 391 (1997) 93, hep-th/9602065.

[22] M. Vasudevan, K. A. Stevens and D. N. Page, "Separability of the Hamilton-Jacobi and Klein-Gordon equations in Kerr-de Sitter metrics," Class. Quant. Grav. 22 (2005) 339, gr-qc/0405125.

[23] B. Carter, "Hamilton-Jacobi and Schrodinger separable solutions of Einstein's equations," Comm. Math. Phys. 10 (1968) 280. 